\title{
Gluon emission at small longitudinal momenta in the QCD effective action approach
}

\author{
M. A. Braun, M. I. Vyazovsky ${ }^{\mathrm{a}}$ \\ Departament of High Energy physics, Saint-Petersburg State University, 198504 St. Petersburg, Russia
}

Received: 25 January 2019 / Accepted: 18 April 2019 / Published online: 7 May 2019

(C) The Author(s) 2019

\begin{abstract}
In the framework of the QCD effective action the vertices of gluon emission in interaction of reggeons are studied in the limit of small longitudinal momenta of the emitted gluon. It is found that the vertices drastically simplify in this limit, so that the gluon becomes emitted from a single reggeon coupled to the projectile and target via multireggeon vertices. The contribution from this kinematical region is studied for double and $2 \times 2$ elementary collisions inside the composite projectile and target.
\end{abstract}

\section{Introduction}

One of the main observables in high-energy collisions of heavy nuclei is the inclusive cross-section for production of secondaries. In the perturbative approach it reduces to production of gluons, which subsequently transform into observed secondary hadrons. The study of gluon production in the central rapidity region with transverse momenta much smaller than the longitudinal momenta of the colliding particles ("Regge kinematics") has a long history, starting from the pioneer work on the production of minijets from the BFKL pomeron [1]. Later this problem was studied in the framework of the dipole picture for the inclusive cross-section in deep-inelastic scattering (DIS) on the heavy nucleus [2], where it was shown that the inclusive crosssection was related to the so-called unintegrated gluon density in the nucleus. Still later in the formalism of reggeized gluons ("BFKL-Bartels" or the BFKLB framework [3-5]) it was demonstrated that the same cross-section consists of a sum of two contributions coming from the BFKL pomeron and the cut triple pomeron vertex [6]. In collisions of two heavy nuclei ("AB collisions") the situation is not so straightforward with the results obtained only in the framework of the JIMWLK formalism (see [7] and the references therein). However, the lack of connection with the actual gluon pro-

a e-mail: ovsky@mv8660.spb.edu duction in a collision of composite targets and the absence of the confirmation in the BFKLB framework leave certain open questions, which are waiting for their solution.

In the BFKLB framework gluon production is based on the vertices obtained by the dispersive technique which uses multiple discontinuities at poles corresponding to intermediate particles. Such vertices depend only on the transversal momenta of gluons and reggeons. In this form the relation to the scattering on composites is poorly understood. To describe it one rather has to study the vertices with the dependence on all 4-momenta included, both transversal and longitudinal. Such vertices can be found by means of the Lipatov Effective Action (LEA) for QCD [8], which introduces the reggeons as independent dynamical variables and describes their interaction with the gluons apart from the standard QCD action. The simplest vertex for gluon emission from a single reggeon $\Gamma_{\mathrm{R} \rightarrow \mathrm{RG}}$ was constructed in the original BFKL paper $[3,4]$. In our previous papers we have found higher vertices for gluon production in the interaction of one and two reggeons, $\Gamma_{\mathrm{R} \rightarrow \mathrm{RRG}}, \Gamma_{\mathrm{R} \rightarrow \mathrm{RRRG}}$ and $\Gamma_{\mathrm{RR} \rightarrow \mathrm{RRG}}$. They are quite complicated and the derivation of the full inclusive cross-section in hA and especially AA collisions seems to require an extraordinary effort, also taking into account that apart from the contribution from the vertices one has to calculate numerous contributions from rescattering.

One has to take into account that LEA only describes the interaction of gluons and reggeons at a given rapidity $y$ (or rather within a finite interval of rapidities $\Delta y$ ). Slices of the total rapidity region separated by large rapidity intervals interact via the exchange of reggeons. So one has to divide the total rapidity into different number of large intervals connecting different slices of effective action and one obtains different diagrams made of effective vertices and reggeon propagators. Neglecting the restriction imposed on the width of each slice may lead to divergencies in the integrals over rapidities in the loop integrals. The practical realization of this picture was first achieved by the separation from the 
whole $y$-integration parts of small intervals $\Delta y$ in the calculation of the NLO BFKL kernel in [9] where clusters of real gluons were produced in the intermediate state. Later it was found that in loop calculations the introduction of such a direct slicing in rapidity by $\Delta y$ was inconvenient. Instead a different method to avoid divergencies due to the limited validity of the LEA vertices was developed based on the so-called tilted Wilson lines (or tilted light-cone variables). Within this method all different divergencies coming from the loop integrations in vertices and propagators cancel [1013].

Vertices studied in this paper refer to a fixed value of rapidity. They do not contain internal integrations nor any divergencies. So their calculation can be safely done within the rules of LEA for a given rapidity, without any cutoff $\Delta y$ or tilted light-cone technique. We shall find that when the longitudinal momenta of the emitted gluon become small our vertices acquire a simple limiting form, which actually corresponds to quite different diagrams of the LEA containing the internal reggeon exchange. These limiting diagrams do not appear in LEA normally and exist only as limits of the standard contributions. However, their simple structure greatly simplifies the calculation of the contribution to the cross-section in the limiting domain of momenta of the emitted particles.

The bulk of the paper is devoted to the behavior of the production vertices at small longitudinal moments (Sects. 2 and 3). In Sect. 4 we study the double inclusive cross-section in the kinematic region where it is determined by the degenerate vertices found. Section 5 contains conclusions and some discussion.

\section{Vertices $\Gamma_{\mathbf{R} \rightarrow \mathrm{RRG}}$ and $\Gamma_{\mathrm{R} \rightarrow \mathrm{RRRG}}$ at small $p_{-}$}

To clearly see the problem we start with the simplest ("Lipatov") vertex $\Gamma_{R \rightarrow R G}$ for the production of the gluon from a reggeon, shown in Fig. 1. The incoming reggeon with momentum $q$ has $q_{+}=p_{+}$and $q_{-}=0$, the outgoing reggeon with momentum $r$ has $r_{+}=0$ and $r_{-}=-p_{-}$.

Fig. 1 Vertex $\Gamma_{R \rightarrow R G}$

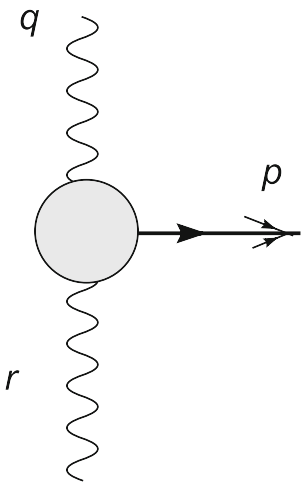

As a result the energy square for the vertex $s_{0}=(q-r)^{2}=$ $p^{2}=0$ and partial energies $s_{1}=(q-p)^{2}$ and $s_{2}=(p+r)^{2}$ have orders $p_{\perp}^{2}$ considered small relative to the total energy squared $s\left(\left|p_{\perp}\right|^{2} / s<<1\right)$. This means that the vertex has a finite dimension in rapidity characterized by the logarithm of these partial energies.

Now consider a more complicated vertex $\Gamma_{R \rightarrow R R G}$ which corresponds to the production of the gluon when a single reggeon goes into two reggeons. This vertex enters the amplitude for the gluon production on a composite state composed of two elementary targets, as we shall see later. The vertex $\Gamma_{\mathrm{R} \rightarrow \mathrm{RRG}}$ is illustrated in Fig. 2a. It was derived in the effective action approach in $[15,16]$ :

$\Gamma_{\mathrm{R} \rightarrow \mathrm{RRG}}=W+R+(1 \leftrightarrow 2)$

Here

$$
\begin{aligned}
& W=-i f^{a b_{1} c} f^{c b_{2} d} \frac{2 q_{+} q^{2}}{\left(q-r_{1}\right)^{2}+i 0} B\left(p, r_{2}, r_{1}\right), \\
& R=i f^{a b_{1} c} f^{c b_{2} d} \frac{q^{2}}{r_{1-}} L\left(p, r_{2}\right) .
\end{aligned}
$$

Notations for momenta are indicated in Fig. 2a; $L$ and $B$ are the Lipatov and Bartels vertices,

$$
\begin{aligned}
L\left(p, r_{2}\right) & =\frac{(p e)_{\perp}}{p_{\perp}^{2}}-\frac{\left(p+r_{2}, e\right)_{\perp}}{\left(p+r_{2}\right)_{\perp}^{2}}, \\
B\left(p, r_{2}, r_{1}\right) & =L\left(p+r_{2}, r_{1}\right) .
\end{aligned}
$$
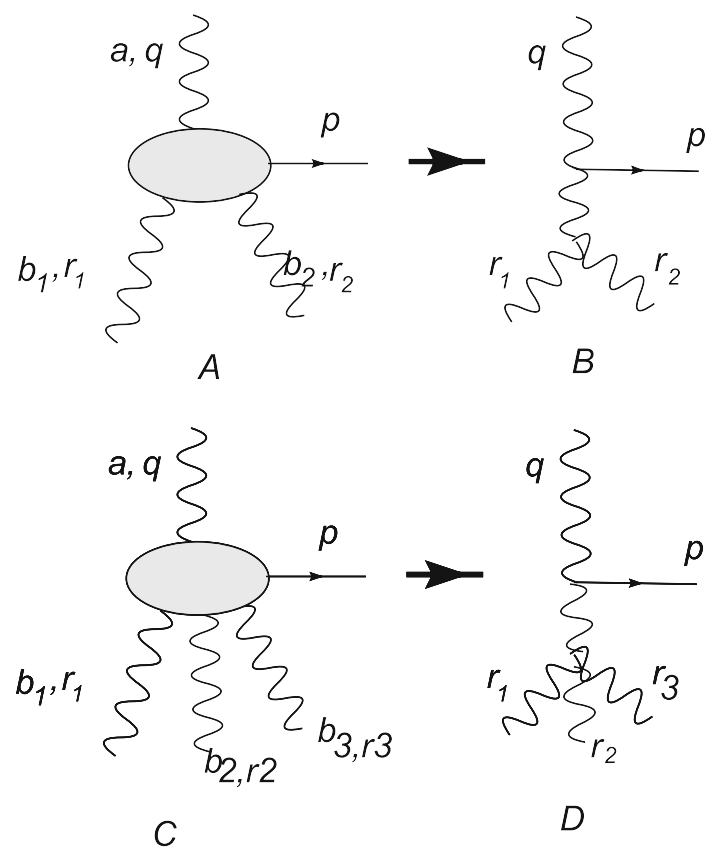

Fig. 2 Reduction of the vertices $R \rightarrow R R G$ and $R \rightarrow R R R G$ for the emission of a particle (gluon) with a small "-" component of its momentum 
Here $e$ is the polarization 4-vector with $e_{+}=0$. Finally, ( $1 \leftrightarrow 2$ ) means the interchange of outgoing reggeons.

We are going to study the vertex in the limiting region $p_{-}<<r_{1,2-}$ (region of small $p_{-}$). In this case $r_{1-} \simeq-r_{2-}$, so that the "-" momenta of the two outgoing reggeons are large and opposite. The '-' momentum comes along one of the outgoing reggeons and goes back along the other.

The part $W$ contains a pole at $\left(q-r_{1}\right)^{2} \simeq-q_{+} r_{1-}=0$ that is at $r_{2-}=p_{-}=\epsilon$. Its contribution to the vertex contains $\delta\left(p_{-}-r_{2-}\right)=\delta\left(p_{-}-\epsilon\right)$. It is this contribution which is the only one taken into account in the BFKLB approach, in which discontinuities in energies are studied. As we move to lower values of $p_{-}<<r_{2}$ this $\delta$ function disappears and only the principal value part of the pole remains. So at $p_{-}<<\left|r_{1,2-}\right|$ the denominator of (2) simplifies as

$$
\begin{aligned}
\left(q-r_{1}\right)^{2} & =\left(p+r_{2}\right)^{2}=r_{2}^{2}+2\left(p r_{2}\right)_{\perp}+2 p_{+} r_{2-} \\
& =r_{2}^{2}+2\left(p r_{2}\right)_{\perp}-p_{\perp}^{2} \frac{r_{2-}}{p_{-}} .
\end{aligned}
$$

Since $r_{2}^{2}=r_{2 \perp}^{2}$ and $p_{\perp}$ are assumed to have the same order of magnitude characteristic for all transverse momenta, at $p_{-}<<\left|r_{2-}\right|$ one can neglect the first two terms, so that $W$ becomes

$$
\begin{aligned}
W & =i f^{a b_{1} c} f^{c b_{2} d} \frac{2 q_{+} q^{2} p_{-}}{r_{2-} p_{\perp}^{2}} B\left(p, r_{2}, r_{1}\right) \\
& =-i f^{a b_{1} c} f^{c b_{2} d} \frac{q^{2}}{r_{2}} B\left(p, r_{2}, r_{1}\right),
\end{aligned}
$$

where we used $q_{+}=p_{+}$and $2 p_{+} p_{=-} p_{\perp}^{2}$. Finally, at $p_{-}<<$ $\left|r_{1,2-}\right|$ we have $r_{1-}+r_{2-}=0$ and

$W=i f^{a b_{1} c} f^{c b_{2} d} \frac{q^{2}}{r_{1-}} B\left(p, r_{2}, r_{1}\right)$.

In the sum $W+R$ we find

$W+R=i f^{a b_{1} c} f^{c b_{2} d} \frac{q^{2}}{r_{1-}}\left(B\left(p, r_{2}, r_{1}\right)+L\left(p, r_{2}\right)\right)$.

We have

$$
\begin{aligned}
& B\left(p, r_{2}, r_{1}\right)+L\left(p, r_{2}\right)=\frac{\left(p+r_{2}, e\right)_{\perp}}{\left(p+r_{2}\right)_{\perp}^{2}}-\frac{\left(p+r_{1}+r_{2}, e\right)_{\perp}}{\left(p+r_{1}+r_{2}\right)_{\perp}^{2}} \\
& +\frac{(p e)_{\perp}}{p_{\perp}^{2}}-\frac{\left(p+r_{2}, e\right)_{\perp}}{\left(p+r_{2}\right)_{\perp}^{2}}=L\left(p, r_{1}+r_{2}\right),
\end{aligned}
$$

so that

$W+R=i f^{a b_{1} c} f^{c b_{2} d} \frac{q^{2}}{r_{1-}} L\left(p, r_{1}+r_{2}\right)$.

Adding $(1 \leftrightarrow 2)$ we get

$$
\Gamma_{\mathrm{R} \rightarrow \mathrm{RRG}}=i \frac{q^{2}}{r_{1-}} L\left(p, r_{1}+r_{2}\right)\left(f^{a b_{1} c} f^{c b_{2} d}-f^{a b_{2} c} f^{c b_{1} d}\right),
$$

where we once again used $r_{2-}=-r_{1-}$. Using the Jacoby identity

$f^{a b_{1} c} f^{c b_{2} d}-f^{a b_{2} c} f^{c b_{1} d}=f^{d a c} f^{c b_{1} b_{2}}$

we finally obtain

$\Gamma_{\mathrm{R} \rightarrow \mathrm{RRG}}=i f^{d a c} f^{c b_{1} b_{2}} \frac{q^{2}}{r_{1-}} L\left(p, r_{1}+r_{2}\right)$.

This expression corresponds to the reduction of the vertex $\Gamma_{\mathrm{R} \rightarrow \mathrm{RRG}}$ as illustrated in the upper part of Fig. 2 .

The diagram Fig. $2 b$ by itself does not appear in LEA. The central reggeon in it has both its two components of the longitudinal momentum equal to zero and so this diagram lies outside the standard Regge kinematics. It only appears as a certain limit of the perfectly legitimate diagram Fig. 2a. One may indicate some arguments to understand its appearance. The initial diagram, Fig. 2a, refers to a given rapidity $y$. The coupled reggeons correspond to virtual particles and cannot be characterized by their rapidities by themselves. However, we can instead consider different partial energies spanned by the interacting gluon and reggeons: the overall energy $s_{0}=\left(q-r_{1}-r_{2}\right)^{2}=p^{2}=0$ and also $s_{1}=\left(q-r_{1}\right)^{2}$ and $s_{2}=\left(q-r_{2}\right)^{2}$. Then $s_{1}=-q_{+} r_{1-}+\left(q-r_{1}\right)_{\perp}^{2}$, where we take into account that $q_{-}=r_{1,2+}=0$. Since $q_{+}=p_{+}=$ $-p_{\perp}^{2} / 2 p_{-}$, we have

$s_{1}=\frac{r_{1-}}{2 p_{-}} p_{\perp}^{2}+\left(q-r_{1}\right)_{\perp}^{2}$.

This energy squared is finite if $p_{-}$and $r_{1-}$ have the same order. In this case the vertex has a finite dimension in energy. The same is true for the energy squared, $s_{2}$. However, if $p_{-} \rightarrow 0$ energies $s_{1}$ and $s_{2}$ become large, then one may think that this is equivalent to a large interval in rapidity covered by the vertex in Fig. 2a, which appears outside the allowed region in LEA. According to this logic in this region one has to divide this interval in two large ones connected by the reggeon. This will bring us to the diagram with two local vertices shown in Fig. 2b. Continuing with this logic one is supposed to introduce a rapidity cutoff $\Delta y$, insert it in some way into the diagrams, and for small $p_{-}$give up the diagram of Fig. 2a and use instead the diagram with the intermediate reggeon and two vertices of Fig. 2b. However, this logic is in fact dubious. There seems to be no clear way to introduce a cutoff $\Delta y$ into the diagram Fig. $2 \mathrm{a}$ and the diagram Fig. $2 \mathrm{~b}$ does not exist in LEA. So the diagram Fig. 2 a can be safely used for any values of $p_{-}$, and Fig. $2 \mathrm{~b}$ emerges only as its limit.

The same phenomenon occurs with the vertex $\Gamma_{R} \rightarrow$ RRRG for gluon emission in the splitting of a reggeon into three reggeons, which is demonstrated in detail in Sect. 3.6 of [17]. In the kinematics $p_{-}<<r_{1-}, r_{2-}, r_{3-}$ it reduces to

$$
\Gamma_{\mathrm{R} \rightarrow \mathrm{RRRG}}=g^{3}\left(f^{a d c} f^{c b_{1} b} f^{b b_{3} b_{2}} \frac{1}{r_{1-}\left(r_{1-}+r_{2-}\right)}\right.
$$




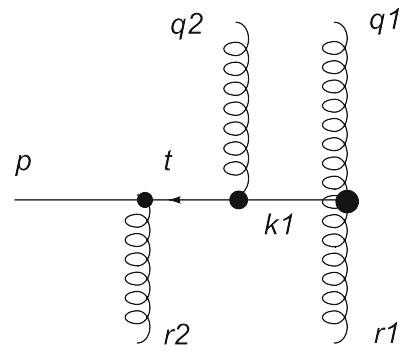

A

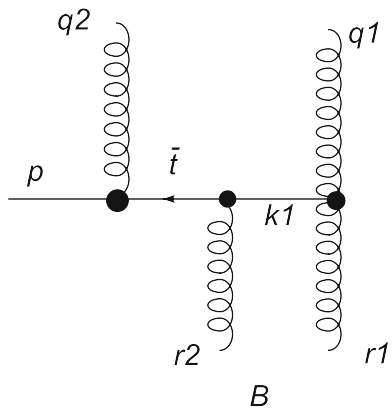

B
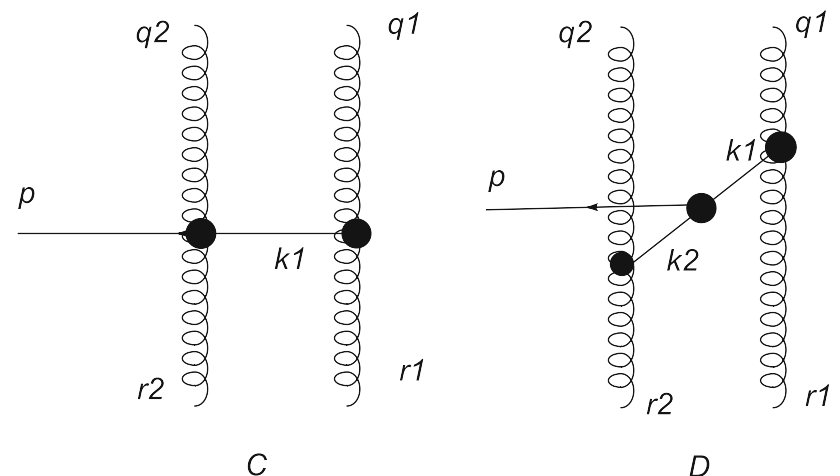

Fig. 3 Diagrams for $\Gamma_{\mathrm{RR}} \rightarrow \mathrm{RRG}$

+ permutations of $r_{1,2}$ and $\left.b_{1,2}\right) L\left(p, r_{1}+r_{2}+r_{3}\right)$,

which is illustrated in the lower part of Fig. 2.

\section{Vertex RR $\rightarrow$ RRG at small $p_{ \pm}$}

The vertex $\mathrm{RR} \rightarrow \mathrm{RRG}$ in the general kinematics was derived in [18]. It is much more complicated than the vertices $\mathrm{R} \rightarrow$ $\mathrm{RRG}$ and $\mathrm{R} \rightarrow \mathrm{RRRG}$.

We shall demonstrate that at small $p_{ \pm}$the same reduction holds for this vertex as for $\Gamma_{\mathrm{R}} \rightarrow \mathrm{RRG}$ and $\Gamma_{\mathrm{R}} \rightarrow \mathrm{RRRG}$ discussed in the previous section, which drastically simplifies the vertices expressing them via the simple BFKL vertex $\Gamma_{\mathrm{R} \rightarrow \mathrm{RG}}$ and multigluon vertices. This reduction is illustrated in Fig. 11.

The vertex $\mathrm{RR} \rightarrow \mathrm{RRG}$ consists of four terms, which are shown in Fig. 3. Explicit expressions for them on the mass shell and multiplied by the polarization vector $\epsilon$ are given in [18]. Below we find expressions for them when

$p_{-}<<\left|r_{1-}\right|,\left|r_{2-}\right|$, and $p_{+}<<\left|q_{1+}\right|,\left|q_{2+}\right|$.

Some technical details can be found in the appendix.

1. Figure $3 a$. We find from this diagram

$$
\begin{aligned}
\Gamma_{1} & =-C_{1} \frac{1}{q_{1+r_{1}-}}\left(e, q_{1}+q_{2}+r_{1}\right)_{\perp} \\
& =-C_{1} \frac{1}{q_{1+} r_{1-}}\left(e, p+2 r_{1}+r_{2}\right)_{\perp} .
\end{aligned}
$$

We recall that here $e$ is the polarization 4-vector with $e_{+}=0$.

2. Figure $3 b$.

From this diagram in the kinematics (13) we find

$$
\begin{aligned}
\Gamma_{2}= & C_{2}\left\{\frac{1}{2}(p e)_{\perp} \frac{1}{p_{+} r_{1-}}-\frac{1}{q_{1+} r_{1-}}\left(e, 2 p-2 q_{1}-q_{2}\right)_{\perp}\right. \\
& \left.-\frac{1}{q_{1+} r_{1-}} \frac{(p e)_{\perp}}{p_{\perp}^{2}}\left(4\left(p, r_{1}+r_{2}\right)_{\perp}+\bar{t}_{\perp}^{2}+2 q_{1}^{2}-q_{2}^{2}+E_{0}\right)\right\} .
\end{aligned}
$$

Here $\bar{t}=p-q_{2}$.

3. Figure $3 \mathrm{c}$.

This diagram generates two terms with different color factors. The corresponding contributions $\Gamma_{3}$ and $\Gamma_{4}$ in the kinematics (13) are given by

$$
\begin{aligned}
\Gamma_{3}= & C_{3}\left\{\frac{1}{p_{+} r_{1-}}(e p)_{\perp}\right. \\
& \left.+\frac{1}{2} \frac{1}{q_{1+} r_{1-}}\left(e, q_{1}+r_{1}\right)_{\perp}+\frac{1}{q_{1+} r_{1-} p_{\perp}^{2}}(e p)_{\perp} q_{2}^{2}\right\}
\end{aligned}
$$

and

$\Gamma_{4}=C_{4}\left\{-\frac{1}{2} \frac{1}{p_{+} r_{1-}}(e p)_{\perp}+\frac{1}{2} \frac{1}{q_{1+} r_{1-}}\left(e, q_{1}+r_{1}\right)_{\perp}\right\}$.

4. Figure 3d.

In the kinematics (13) this diagram gives

$$
\begin{aligned}
\Gamma_{5}= & C_{5}\left\{\frac{1}{2} \frac{(p e)_{\perp}}{p_{+}}\left(\frac{1}{r_{1-}}-\frac{1}{r_{2-}}\right)\right. \\
& \left.-\frac{1}{2 q_{1+} r_{1-}}\left(q_{1}-r_{1}-q_{2}+r_{2}, e\right)_{\perp}\right\} .
\end{aligned}
$$

The color factors are

$C_{1}=f^{d b_{2} c} f^{b_{1} a_{1} e} f^{e a_{2} d}, \quad C_{2}=f^{d a_{2} c} f^{b_{1} a_{1} e} f^{d b_{2} e}$, $C_{3}=f^{a_{1} b_{1} d} f^{a_{2} c e} f^{b_{2} d e}, C_{4}=f^{a_{1} b_{1} d} f^{a_{2} d e} f^{b_{2} c e}$, $C_{5}=f^{a_{1} d_{1} b_{1}} f^{d_{1} c d_{2}} f^{a_{2} d_{2} b_{2}}$,

so that

$C_{3}=-C_{2}, \quad C_{4}=C_{1}$

and using the Jacoby identity

$C_{5}=C_{4}-C_{3}=C_{1}+C_{2}$.

These expressions are to be symmetrized over permutations of reggeons. Since $\Gamma_{1}, \ldots, \Gamma_{4}$ are to be symmetrized over all permutations of momenta and colors of the two 
incoming and two outgoing reggeons and $\Gamma_{5}$ only over the two outgoing reggeons, we can totally symmetrize the sum of all diagrams taking the contribution from $\Gamma_{5}$ with weight $1 / 2$.

First we show that terms of the leading order $1 /\left(p_{+} r_{1-}\right)$ and $1 /\left(p_{+} r_{2-}\right)$ cancel. Before symmetrization they are

$$
\begin{aligned}
C_{1} & {\left[-\frac{(p e)_{\perp}}{2 p_{+} r_{1-}}+\frac{1}{2} \frac{(p e)_{\perp}}{2 p_{+}}\left(\frac{1}{r_{1-}}-\frac{1}{r_{2-}}\right)\right] } \\
& +C_{2}\left[\frac{(p e)_{\perp}}{2 p_{+} r_{1-}}-\frac{(p e)_{\perp}}{p_{+} r_{1-}}+\frac{1}{2} \frac{(p e)_{\perp}}{2 p_{+}}\left(\frac{1}{r_{1-}}-\frac{1}{r_{2-}}\right)\right] \\
= & C_{1}\left[-\frac{(p e)_{\perp}}{4 p_{+}}\left(\frac{1}{r_{1-}}+\frac{1}{r_{2-}}\right)\right]+C_{2}\left[-\frac{(p e)_{\perp}}{4 p_{+}}\left(\frac{1}{r_{1-}}+\frac{1}{r_{2-}}\right)\right] \\
= & -\frac{1}{4}\left(C_{1}+C_{2}\right)\left[\frac{(p e)_{\perp}}{p_{+} r_{1-}}+\frac{(p e)_{\perp}}{p_{+} r_{2-}}\right] .
\end{aligned}
$$

At all permutations the momentum factor in (21) does not change. So the leading order contribution is determined by the symmetrized combinations of the color factors. One has

$$
\begin{aligned}
\operatorname{Sym}\left\{C_{1}+C_{2}\right\}= & \operatorname{Sym}\left\{C_{5}\right\}=C_{5}+C_{5}\left(a_{1} \leftrightarrow a_{2}\right) \\
& +C_{5}\left(b_{1} \leftrightarrow b_{2}\right)+C_{5}\left(a_{1} \leftrightarrow a_{2}, b_{1} \leftrightarrow b_{2}\right)=0 .
\end{aligned}
$$

Thus the leading order contribution is indeed canceled.

Next we study the factor multiplying $1 /\left(q_{1+} r_{1-}\right)$. First we address separate terms which do not contain $(p e)_{\perp} / p_{\perp}^{2}$ :

$$
\begin{aligned}
- & C_{1}\left(e, p+2 r_{1}+r_{2}\right)_{\perp}-C_{2}\left(e, 2 p-2 q_{1}-q_{2}\right)_{\perp} \\
& -\frac{1}{2} C_{2}\left(e, q_{1}+r_{1}\right)_{\perp}+\frac{1}{2} C_{1}\left(e, q_{1}+r_{1}\right)_{\perp} \\
& -\frac{1}{2}\left(C_{1}+C_{2}\right)\left(e, q_{1}-q_{2}-r_{1}+r_{2}\right)_{\perp} \\
= & \left(-C_{1}+C_{2}\right)\left(e, q_{1}+q_{2}\right)_{\perp}-2 C_{2}(p e)_{\perp} \\
& +\frac{1}{4}\left(C_{1}+C_{2}\right)(p e)_{\perp} .
\end{aligned}
$$

Then we transform the factor multiplying the product of $1 /\left(q_{1+} r_{1-}\right)$ and $(p e)_{\perp} / p_{\perp}^{2}$ (using $\left.\bar{t}=q_{1}-r_{1}-r_{2}\right)$ :

$$
\begin{aligned}
& -C_{2}\left(4\left(p, r_{1}+r_{2}\right)_{\perp}+\bar{t}_{\perp}^{2}+2 q_{1}^{2}-q_{2}^{2}+E_{0}\right)-C_{2}\left(q_{2}^{2}\right) \\
& =-2 C_{2}\left(p+r_{1}+r_{2}\right)_{\perp}^{2}+2 C_{2} p_{\perp}^{2} .
\end{aligned}
$$

As a result, the total factor multiplying $1 /\left(q_{1+} r_{1-}\right)$ takes the form [using $p+r_{1}+r_{2}=q_{1}+q_{2}$ and noting that the second term in (23) cancels the second term in (24)]

$$
\begin{aligned}
& \left(-C_{1}+C_{2}\right)\left(e, q_{1}+q_{2}\right)_{\perp}-2 C_{2} \frac{(p e)_{\perp}}{p_{\perp}^{2}}\left(q_{1}+q_{2}\right)_{\perp}^{2} \\
& +\frac{1}{4}\left(C_{1}+C_{2}\right)(p e)_{\perp} .
\end{aligned}
$$

Now we pass to symmetrization. Taking the order of the two incoming and two outgoing reggeons as $\Gamma_{i} \equiv$ $\Gamma_{i}(2,1 \mid 2,1)$ in the expressions for $\Gamma_{i}, i=1, \ldots, 5$ we have for the total vertex

$$
\sum_{i=1}^{5}\left[\Gamma_{i}(2,1 \mid 2,1)+\Gamma_{i}(1,2 \mid 2,1)+\Gamma_{i}(2,1 \mid 1,2)+\Gamma_{i}(1,2 \mid 1,2)\right] .
$$

The transverse factors in (25) do not change under permutations. Since in our kinematics with the adopted precision $q_{2+}=-q_{1+}$ and $r_{2-}=-r_{1-}$, the denominator $q_{1+} r_{1-}$ changes sign for the reggeon configurations $(1,2 \mid 2,1)$ or $(2,1 \mid 1,2)$ and does not change sign for the configuration $(1,2 \mid 1,2)$. As a result we find antisymmetric combinations of the color factors,

$$
\begin{aligned}
\operatorname{Asym}\left\{C_{i}\right\}= & C_{i}-C_{i}\left(a_{1} \leftrightarrow a_{2}\right)-C_{i}\left(b_{1} \leftrightarrow b_{2}\right) \\
& +C_{i}\left(a_{1} \leftrightarrow a_{2}, b_{1} \leftrightarrow b_{2}\right) .
\end{aligned}
$$

Take first $C_{5}=f^{a_{1} b_{1} d} f^{d c e} f^{e a_{2} b_{2}}$ :

$$
\begin{aligned}
\operatorname{Asym}\left\{C_{5}\right\}= & \operatorname{Asym}\left\{C_{1}+C_{2}\right\} \\
= & f^{a_{1} b_{1} d}\left(f^{d c e}+f^{e c d}\right) f^{e a_{2} b_{2}} \\
& -f^{a_{2} b_{1} d}\left(f^{d c e}+f^{e c d}\right) f^{e a_{1} b_{2}}=0,
\end{aligned}
$$

from which it follows that Asym $\left\{C_{1}\right\}=-\operatorname{Asym}\left\{C_{2}\right\}$.

As a result the last term in (25) gives no contribution to the total vertex. It takes the form

$$
\frac{\operatorname{Asym}\left\{C_{2}\right\}}{q_{1+} r_{1-}}\left(2\left(e, q_{1}+q_{2}\right)_{\perp}-2 \frac{(p e)_{\perp}}{p_{\perp}^{2}}\left(q_{1}+q_{2}\right)_{\perp}^{2}\right) .
$$

To calculate

$$
\begin{aligned}
\operatorname{Asym}\left\{C_{2}\right\}= & f^{a_{1} b_{1} d} f^{d b_{2} e} f^{e a_{2} c}-f^{a_{2} b_{1} d} f^{d b_{2} e} f^{e a_{1} c} \\
& -f^{a_{1} b_{2} d} f^{d b_{1} e} f^{e a_{2} c}+f^{a_{2} b_{2} d} f^{d b_{1} e} f^{e a_{1} c},
\end{aligned}
$$

one has to apply the Jacoby identity three times. First consider the difference between the first and third terms. Applying

$$
f^{a_{1} b_{1} d} f^{d b_{2} e}+f^{b_{1} b_{2} d} f^{d a_{1} e}+f^{b_{2} a_{1} d} f^{d b_{1} e}=0
$$

it can be rewritten as $-f^{b_{1} b_{2} d} f^{d a_{1} e} f^{e a_{2} c}$. Similarly the difference between the fourth and second terms can be rewritten as $-f^{b_{2} b_{1} d} f^{d a_{2} e} f^{e a_{1} c}$. Finally, one more Jacoby identity allows one to find

$$
\text { Asym } \begin{aligned}
\left\{C_{2}\right\} & =f^{b_{1} b_{2} d}\left(f^{d a_{2} e} f^{e a_{1} c}-f^{d a_{1} e} f^{e a_{2} c}\right) \\
& =-f^{a_{1} a_{2} e} f^{e c d} f^{d b_{1} b_{2}} .
\end{aligned}
$$

The final result for the total vertex is

$$
\Gamma=\frac{f^{a_{1} a_{2} e} f^{e c d} f^{d b_{1} b_{2}}}{q_{1+} r_{1-}}\left(-2\left(e, q_{1}+q_{2}\right)_{\perp}+2 \frac{(p e)_{\perp}}{p_{\perp}^{2}}\left(q_{1}+q_{2}\right)_{\perp}^{2}\right),
$$



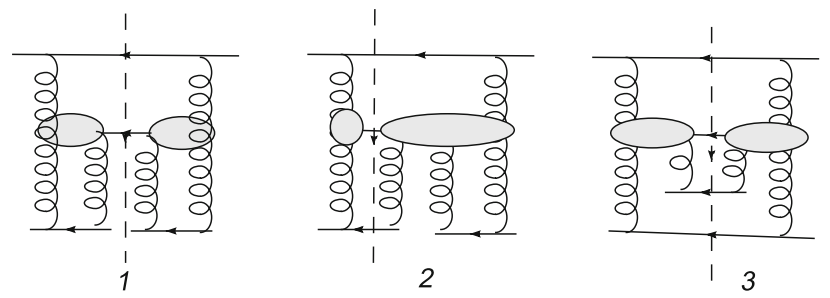

Fig. 4 Cut amplitudes with vertices $\Gamma_{R \rightarrow R R G}$ and $\Gamma_{R \rightarrow R R R G}$
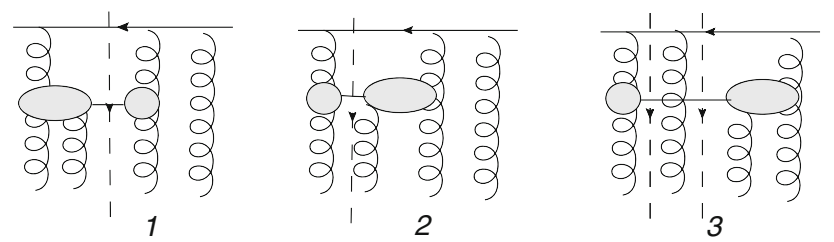

Fig. 5 Typical cut amplitudes with vertex $\Gamma_{R \rightarrow R R G}$ and rescattering

which can be rewritten as

$$
\begin{aligned}
\Gamma_{\mathrm{RR} \rightarrow \mathrm{RRG}}= & 2 f^{a_{1} a_{2} e} f^{e c d} f^{d b_{1} b_{2}} \\
& g^{3}\left(q_{1}+q_{2}\right)_{\perp}^{2} L\left(p, r_{1}+r_{2}\right) \frac{1}{q_{1+} r_{1-}} .
\end{aligned}
$$

\section{The inclusive cross-sections in $\mathrm{hA}$ and $\mathrm{AA}$ collisions at small $p_{ \pm}$}

\section{1 hA collisions}

As we have seen the vertices for gluon production in the interaction of reggeons drastically simplify in the region of small longitudinal momenta of the produced gluon. At first sight it promises to facilitate calculation of the physical observables, such as inclusive cross-sections in the collision of composite particles, e.g. deuterons. However, we shall discover that while such a facilitation certainly takes place, the resulting cross-sections vanish in this region.

We start with hA collisions. We recall that the inclusive cross-section from the double scattering of an elementary projectile is given by the formula

$$
\begin{aligned}
I_{A}(p, y) & \equiv \frac{2(2 \pi)^{3} \mathrm{~d} \sigma}{{\mathrm{d} y \mathrm{~d}^{2} p \mathrm{~d}^{2} b}^{4}} \\
& =\frac{A(A-1)}{4 \pi k_{+} s} \int \mathrm{d} z_{1} \mathrm{~d} z_{2} \mathrm{~d} \epsilon \cos \left(\epsilon m\left(z_{1}-z_{2}\right) / k_{+}\right)
\end{aligned}
$$

$$
\operatorname{Im} H(p, \epsilon) \rho\left(b, z_{1}\right) \rho\left(b, z_{2}\right),
$$

where $s=2 k_{+}^{2}$ is the c.m. energy squared, $\rho(b, z)$ is the nuclear density, $H(p, \epsilon)$ is the high-energy part of the amplitude left after separating the nuclear factor, $p$ is the momentum of the emitted particle (gluon) and $\epsilon$ is the "-" component of the momentum transferred to one of the scattering centers, both in the c.m. system. The naive Glauber approximation follows if

$\operatorname{Im} H=2 \pi \delta(\epsilon) F(p)$.

In this case one gets

$I_{A}(p, y)=\frac{A(A-1)}{2 k_{+} S} T^{2}(b) F(p)$.

The high-energy part $H$ can be found from the scattering amplitude cut to select the observed emitted gluon in the intermediate state. Due to the famous AGK cancelations the gluon can either be emitted from the incoming pomeron or from the cut triple pomeron vertex. The emission from the pomeron is well known. Here we are interested in the emission from the cut triple pomeron vertex which contains the convolutions of vertices $\Gamma_{\mathrm{R} \rightarrow \mathrm{RG}}, \Gamma_{\mathrm{R} \rightarrow \mathrm{RRG}}$ and $\Gamma_{\mathrm{R} \rightarrow \mathrm{RRRG}}$ shown in Fig. 4. Apart from this contribution which comes exclusively from the production vertices $\Gamma$, the high-energy part includes numerous diagrams where one or two reggeons do not interact ("rescattering contribution") illustrated in Figs. 5 and 6. In these figures only typical diagrams are shown. We also do not indicate how the outgoing reggeons are coupled to the two colorless targets. This coupling may be different and follows the pattern of Fig. 4.

We also do not show explicitly evolution of the pomerons attached to the projectile and target (which is well known and standardly realized by the BFKL equation) nor the actual coupling to colorless scattering centers in the nucleus (in fact nucleons). One has to take into account that for the heavy nucleus the two centers have to refer to different nucleons. Otherwise the contribution is down by $A^{1 / 3}$, assumed large. For clarity we show some diagrams with the nuclear target explicitly indicated in Fig. 7. Several cuts in Figs. 5 and 6 imply that the sum over the contribution of each cut should be taken. Our aim is to study these contributions in the limit when the longitudinal momentum $p_{-}$of the created gluon is much smaller than the "-"-momentum $\epsilon$ transferred to the

Fig. 6 Typical cut amplitudes with pure rescattering

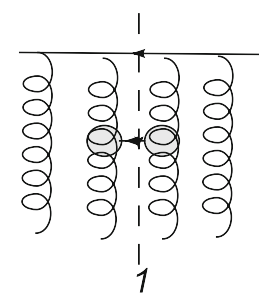

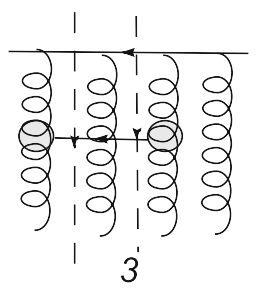

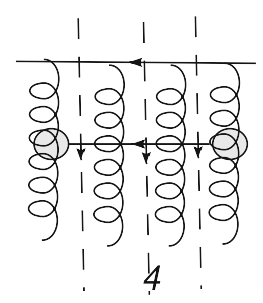



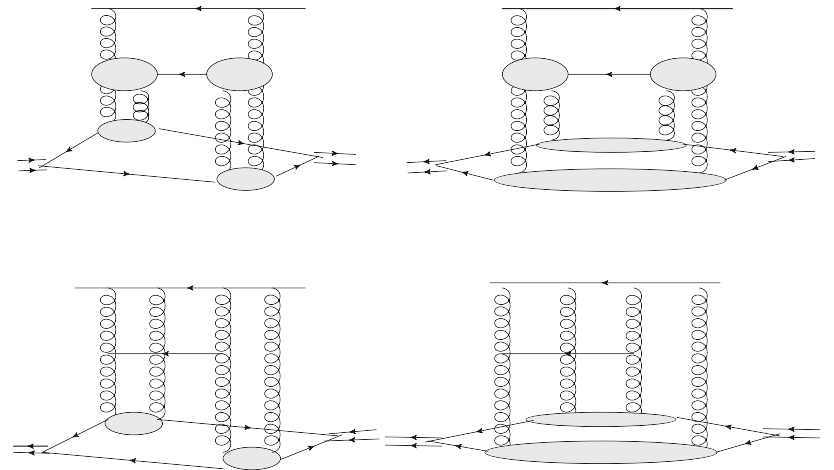

Fig. 7 Typical amplitudes with the coupling to the nuclear target explicitly shown

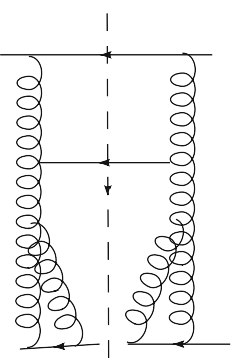

1

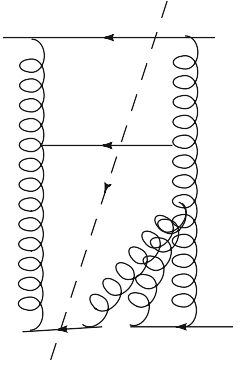

2

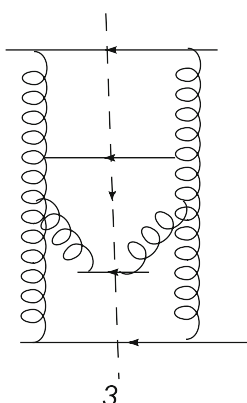

$3^{\prime}$
Fig. 8 Cut amplitudes with vertices $\Gamma_{R \rightarrow R R G}$ and $\Gamma_{R \rightarrow R R R G}$ after reduction in Fig. 2
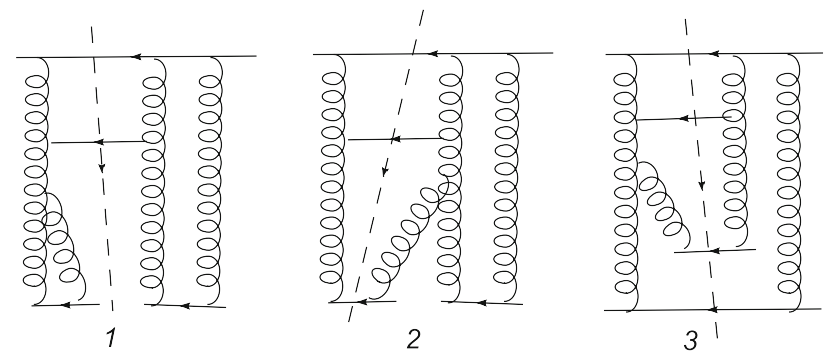

Fig. 9 Cut amplitudes with vertex $\Gamma_{\mathrm{R} \rightarrow \mathrm{RRG}}$ and rescattering after reduction in Fig. 2

target, which is the only dimensional variable after the integration over the longitudinal momenta of the reggeons.

As we have shown, in this kinematics the vertices $\Gamma_{R \rightarrow R R G}$ and $\Gamma_{\mathrm{R} \rightarrow \mathrm{RRRG}}$ degenerate into the simple expressions (11) and (12), illustrated in Fig. 2. The diagrams in Figs. 4 and 5 then transform into Figs. 8 and 9.

Leaving the discussion of the rescattering contribution to the end of this section we concentrate here on the diagrams with the reduced vertices $\Gamma$ in Fig. 8. Since the pomeron vanishes when the two reggeons are located at the same point, all diagrams in which the two final reggeons in $\Gamma_{\mathrm{R}} \rightarrow \mathrm{RRG}$ or $\Gamma_{R \rightarrow R R R G}$ are coupled to the same target vanish. So in the domain $p_{-}<<|\epsilon|$ the inclusive cross-section coming from the cut triple pomeron vertex will be given exclusively by diagram 3 in Fig. 8. which corresponds to squaring the vertex $\Gamma_{\mathrm{R} \rightarrow \mathrm{RRG}}$.

Twice the imaginary part of the high-energy amplitude $H$ will be given by the square of the two production amplitudes, each containing the vertex $\Gamma_{R} \rightarrow R R G$. It contains color, energetic, transverse momentum and numerical factors.

The color factor is given by (at large $N_{\mathrm{c}}$ )

$f^{a e_{1} d} f^{d e_{2} a} f^{e_{1} b_{1} b_{2}} f^{b_{1} e_{2} b_{2}}=\left(-N_{\mathrm{c}} \delta_{e_{1} e_{2}}\right)\left(-N_{\mathrm{c}} \delta_{e_{1} e_{2}}\right)=N_{c}^{4}$.

Energetic factors come from three cut quark propagators. From the projectile quark we find $4 k_{+}^{2} / 2 k_{+}=2 k_{+}$. The two target quarks give each $2 l_{-}$. In the total we get $8 k_{+} l_{-}^{2}$. Apart from this we have factors $1 / r_{1-}$ in each of the two vertices $\Gamma_{\mathrm{R} \rightarrow \mathrm{RRG}}$. Taking into account that $r_{1-}$ on the right differs by $\epsilon$ we find a longitudinal integral

$$
J(\epsilon)=\int \frac{\mathrm{d} r_{1-}}{2 \pi} \mathcal{P} \frac{1}{r_{1-}} \cdot \mathcal{P} \frac{1}{r_{1-}-\epsilon},
$$

where $\mathcal{P}$ means the principal value. Calculation gives

$$
\begin{aligned}
J(\epsilon)= & \frac{1}{8 \pi} \lim _{\eta_{1} \rightarrow 0, \eta_{2} \rightarrow 0} \int \mathrm{d} x\left(\frac{1}{x+i \eta_{1}}+\frac{1}{x-i \eta_{1}}\right) \\
& \left(\frac{1}{x-\epsilon+i \eta_{2}}+\frac{1}{x-\epsilon-i \eta_{2}}\right) \\
= & -\frac{i}{4}\left(\frac{1}{\epsilon-i \eta_{1}-i \eta_{2}}-\frac{1}{\epsilon+i \eta_{2}+i \eta_{1}}\right)=\frac{\pi}{2} \delta(\epsilon)
\end{aligned}
$$

and provides us with the factor $(\pi / 2) \delta(\epsilon)$. So actually the diagram is zero unless $\epsilon$ is different from zero. However, with $\epsilon=0$ one cannot realize the kinematics $p_{-}<<|\epsilon|$, since $p_{-}>0$. This means that in fact the diagram of Fig. 8 , 3 vanishes in this kinematics.

Later we shall see that the diagrams with rescattering also give no contribution in this domain.

\subsection{AB collisions}

Passing to $\mathrm{AB}$ collisions the obvious generalization of (36) gives the inclusive cross-section in $2 \times 2$ collisions in the Glauber approximation,

$$
\begin{aligned}
I_{A B}\left(p, b_{1}, b_{2}, y\right) & \equiv \frac{(2 \pi)^{3} \mathrm{~d} \sigma}{\mathrm{d} y \mathrm{~d}^{2} p \mathrm{~d}^{2} b_{1} \mathrm{~d}^{2} b_{2}} \\
& =\frac{A(A-1) B(B-1)}{2 k_{+} 2 l_{-}} F T_{A}^{2}\left(b_{1}\right) T_{B}^{2}\left(b_{2}\right),
\end{aligned}
$$

where $s=2(k l), T_{A, B}(b)$ are the nuclear profile functions and $F$ is related to the high-energy amplitude $H$ with the "." component $\epsilon$ and the "+" component $\lambda$ of the transferred momenta to one of the two target centers and two projectile centers, respectively, in the c.m. system, by

$\operatorname{Im} H=2 \pi \delta(\epsilon) 2 \pi \delta(\lambda) F+\cdots$. 
Fig. 10 Cut amplitudes with two reggeons attached to each participant
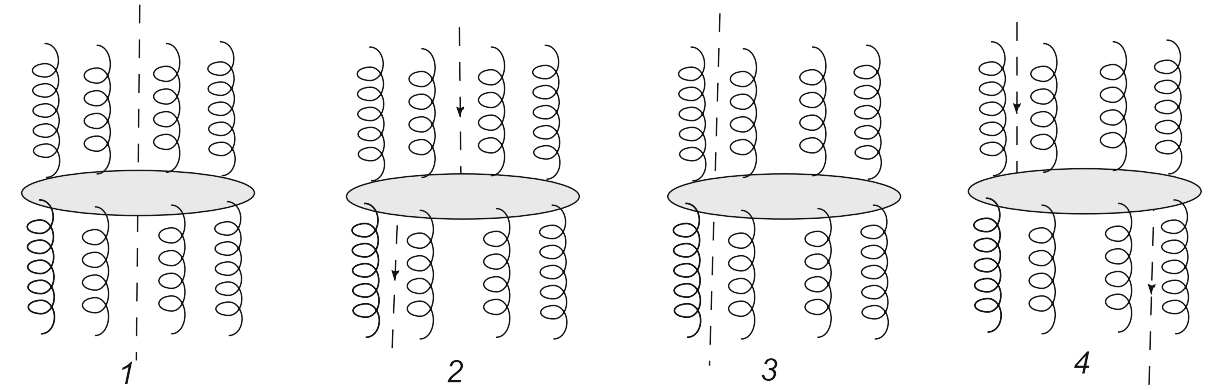

Fig. 11 Reduction of the vertices $R R \rightarrow R R G$, $\mathrm{RR} \rightarrow \mathrm{RRRG}$ and $\mathrm{RRR} \rightarrow$ RRRG for the emission of a particle (gluon) with small \pm components of its momentum

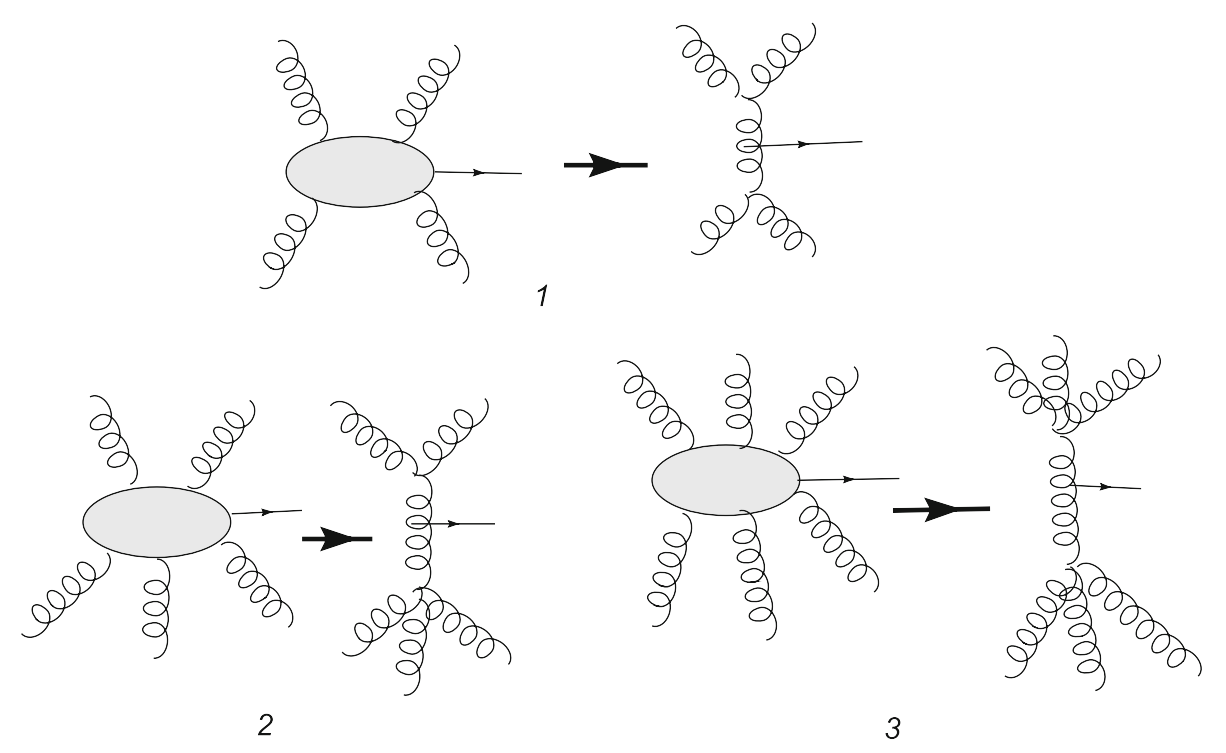

Terms in $H$ which do not contain $\delta(\epsilon) \delta(\lambda)$ give no contribution to the inclusive cross-section.

Due to AGK cancelations [14] we expect that contributions from emission from the cut pomerons cancel and the final result comes exclusively from the cut 4-pomeron interaction vertex, including improper terms corresponding to its disconnected part, corresponding to rescattering in our language. Apart from rescattering and the suppressed evolution each diagram becomes a convolution of two amplitudes for production of the observed gluon in the transition from 1,2 or 3 initial reggeons into 1, 2 or 3 final reggeons. Then graphically $H$ corresponds to the cut diagrams shown in Fig. 10. We do not show the different ways in which the reggeons may be coupled to the two projectiles and targets. They again follow the pattern illustrated in Fig. 4.

The diagrams in Fig. 10 contain first ones with a convolution of two connected vertices for the production of the observed gluon for transition from 1,2 or 3 initial reggeons into 1, 2 or 3 final reggeons. Apart from this there are the diagrams with non-connected vertices, which we interpret as rescattering. We shall consider the kinematic domain of small longitudinal momenta of the emitted gluon,

$p_{-}<<|\epsilon|, \quad p_{+}<<|\lambda|$.
This domain implies small values of $p_{\perp}^{2}$ and so refers to emission in the forward direction. As we shall argue later, the amplitudes with rescattering do not contribute to the crosssection in the domain (41).

As we have previously shown in the domain (13), the vertices $\Gamma_{\mathrm{R} \rightarrow \mathrm{RRG}}, \Gamma_{\mathrm{R} \rightarrow \mathrm{RRRG}}$ and $\Gamma_{R R \rightarrow R R G}$ degenerate into the simple expressions (11), (12) and (33), respectively. Unfortunately we do not know explicit expressions for the production amplitudes $\Gamma_{\mathrm{RR} \rightarrow \mathrm{RRRG}}$ and $\Gamma_{\mathrm{RRR}} \rightarrow \mathrm{RRRG}$. However, in the spirit of the QCD effective action and comparing with cases with smaller numbers of reggeons we firmly believe that also for them a similar reduction takes place. This reduction is graphically shown in the lower part of Fig. 11.

With vertices thus degenerated, the cut diagrams shown in Fig. 10 transform into the diagrams illustrated in Fig. 12. We recall that when the two reggeons forming a pomeron happen to be located at the same point in the coordinate space the corresponding pomeron leg vanishes. This excludes all the diagrams in Fig. 12 except the last.

As we have seen in the kinematics $p_{ \pm} \rightarrow 0$ vertex $\Gamma_{\mathrm{RR} \rightarrow \mathrm{RRG}}$ is simplified to (33). The imaginary part of $H$ will be obtained by squaring this expression together with the relevant energetic, transverse momenta, numerical and color factors. 
Fig. 12 Cut amplitudes for $A B$ scattering with two reggeons attached to each participant and vertices reduced in the kinematics (13)
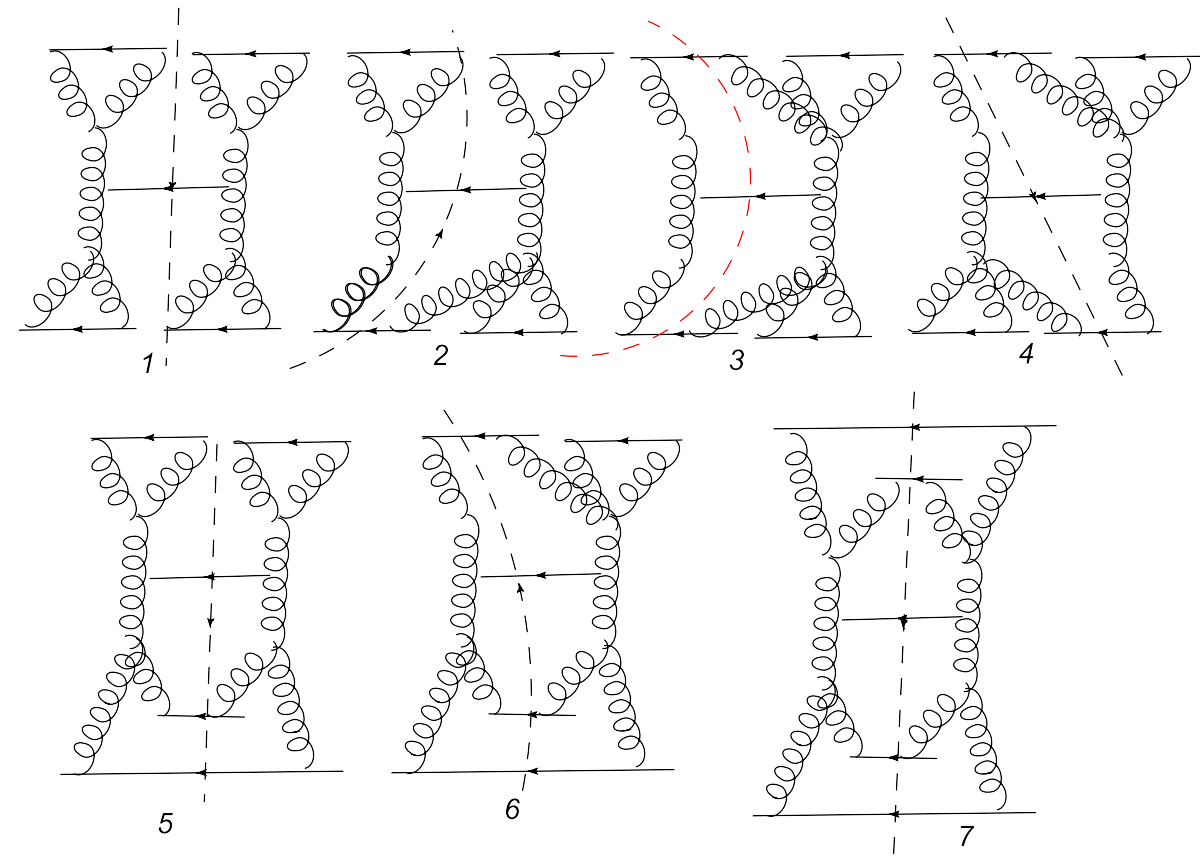

ing diminishes $L$ by 2 . So initially we have $2 M-6-2 R$ integration variables. In the rescattering each quark propagator gives a $\delta$-function restricting one of the longitudinal momenta. The total number of these $\delta$-functions is obviously $M-3$. Two more $\delta$-functions come from the restriction to fix the momentum $p$ of the emitted gluon. As a result, we find

$L=M-2 R-5$.

In the main diagram without rescattering $M=6$, so that $L=1$, which agrees with our previous calculations. The diagrams with a single rescattering have $M=7$ and $R=1$, which gives $L=0$. Finally, with two rescatterings we have $M=8, R=2$ and consequently $L=-1$. This negative $L$ means of course that one $\delta$-function survives after longitudinal integrations. The two remaining longitudinal variables are $p_{-}$and $\epsilon$, so that the result has to be some function of $p_{-}$and $\epsilon$ of dimension -1 .

In the region $p_{-}<<|\epsilon|$ the only remaining variable is $\epsilon$. So in the case of no integration the result has to be $\propto 1 / \epsilon$ and in the case of $L=-1$ it has to be $\propto \delta(\epsilon)$. As we have already mentioned the last case lies outside the assumed kinematics. If the amplitude is $\propto 1 / \epsilon$ it gives zero in (34) as it is odd in $\epsilon$. Calculations also show that in this case the contribution to the amplitude is real and its imaginary part is zero. So rescattering amplitudes do not contribute to the inclusive hA cross-section either.

This argument works also for $\mathrm{AB}$ collisions. In this case the diagrams with rescattering are many and their order in $g$ starts with $g^{10}$ lower than for the main diagram calculated in Section 3. In Fig. 13 we show the diagrams with rescattering of orders $g^{10}$ and $g^{12}$ lower than the order $g^{14}$ considered 


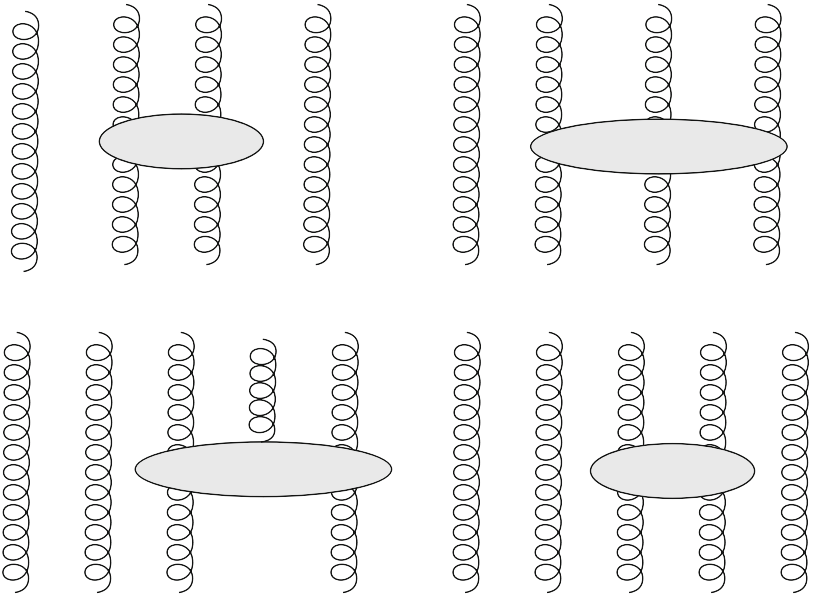

Fig. 13 Diagrams with rescattering of orders $g^{10}$ and $g^{12}$

above. It is trivial to show that they all do not give any contribution to the inclusive cross-section in the domain (13) on the same grounds as for hA scattering.

With four participants and $R$ rescatterings the number of integrations is initially $2(M-4-R)$. Rescattering propagators will produce $M-4$ delta-functions and together with the restriction imposed by fixing $p$ we get in this case

$L=M-2 R-6$.

The order $g^{N}$ of different contributions may be different in this case. It combines the couplings of the reggeons to the participant quarks and the reggeon interactions, supposed to be the only one to correspond to the notion of the vertex (a single fixed rapidity $y$ ). Let the numbers of the incoming and outgoing reggeons be $M_{1}$ and $M_{2}$, respectively. $M=M_{1}+$ $M_{2}$ is their total number. A fraction $R$ of the reggeons may go directly from the projectile to the target (rescatterings). The rest of the reggeons, $M_{1}-R$ from the projectile and $M_{2}-R$ from the target, interact once with the transitional kernel $K_{M_{1}-R \rightarrow M_{2}-R}$, which has order $g^{k}$ with $k=M_{1}+$ $M_{2}-2 R-2=M-2 R-2$. Taking into account the coupling to participants $g^{M}$ we find the total order

$N=2 M-2 R-2$.

One, however, has to take into account the number of interacting incoming and outgoing reggeons-each cannot be smaller than 2. This gives a restriction on the number of rescatterings,

$R \leq M_{1}-2, M_{2}-2$.

Using Eqs. (44) and (45) we find the following diagrams with $R$ rescattering having order $N \leq 14$ which are interesting for our 4-pomeron vertex. We combine the numbers $R$, $N$ and $L$ for the diagram into a single index $(R, N, L)$. We find
1. $M=8:(0,14,2),(1,12,0),(2,10,-2)$;

2. $M=9:(1,14,1),(2,12,-1)$;

3. $M=10:(2,14,0),(3,12,-2)\left(\right.$ only $M_{1}=M_{2}=$ $5)$

4. $M=11:(3,14,-1)$, (only $\left.M_{1}=6, M_{2}=5\right)$;

5. $M=12:(4,14,-2)\left(\right.$ only $\left.M_{1}=M_{2}=6\right)$.

Negative values for $L$ as before imply that one or two $\delta$ functions remain as factors for the diagram.

Inspecting these data we first find our main diagram with $M=8$ and no rescattering. It contains two longitudinal integrations as we have previously seen. We also see that some diagrams with many rescatterings contain no integrations but one or two $\delta$-functions as factors. The final dimension of the longitudinal integral is, however, -2 in all cases.

In the domain (13) the result of the longitudinal integration should be a function of $\epsilon$ and $\lambda$ which is Lorenz-invariant and of dimension -2 . The only candidates are terms proportional to $1 / \epsilon \lambda, \delta(\epsilon) / \lambda, \delta(\lambda) / \epsilon$ and $\delta(\epsilon) \delta(\lambda)$. In all these cases no contribution to the cross-section follows in the domain (13) either because of the oddness in $\epsilon$ or $\lambda$ or because of violating the kinematics.

\section{Conclusions}

The bulk of our paper is devoted to the study of the vertex for transition $R R \rightarrow R R G$ in the special kinematical region where the longitudinal momentum of the emitted gluon is much smaller than the longitudinal momenta of participating reggeons. We were able to show that the vertex drastically simplifies, so that emission proceeds from a single intermediate reggeon connected to the participants via two 3-reggeon vertices. This also reestablishes the role of the 3 -reggeon vertex, absent in many cases due to signature conservation but appearing under certain kinematical conditions. It also indicates the general rule for gluon emission in the interaction of reggeons when the longitudinal momenta of the emitted gluon turn out to be much smaller (larger) than those of the reggeons. Under this condition the emission vertex drastically simplifies from a very complicated general expression to a simple and physically clear form. First examples of such simplification were already mentioned in [16]. Here we found that it remains valid also for the highly complicated vertex $\Gamma_{\mathrm{RR} \rightarrow \mathrm{RRG}}$. We firmly believe that this phenomenon holds also for vertices with any number of incoming and outgoing reggeons.

Our results have a certain significance in the general theory of interacting reggeons within the effective action approach. They refer to the use of the effective action for calculating not only the vertices at a given rapidity but also the amplitudes for physical processes at large overall rapidity. Then according to the idea of effective action one has to divide the total 
rapidity interval in slices of definite intermediate rapidities, which are then connected by reggeon exchanges. It is initially assumed that the vertices determined by the effective action are working only within a given rapidity slice, so that the set of diagrams describing the amplitude depends on the number of divisions of the total rapidity (resolution in rapidity). Our results show that this situation is different: the set of diagrams actually does not depend on the resolution. Taking the lowest resolution and using the appropriate set of diagrams for the intermediate rapidity one automatically obtains a different set of diagrams appropriate for higher resolution when one considers the limiting expressions for the initial resolution. In this sense we prove the independence of the slicing of the whole rapidity into partial intervals as supposed to be true in the original derivation of LEA.

Our proof is not complete and does not cover all possible cases. It is based on the vertices which have been explicitly calculated earlier. In fact the vertices become very complicated with the growth of the number of reggeons and emitted particles. However, we firmly believe that the result obtained for the considered comparatively simple vertices is true in more complicated cases.

As an application we considered the contribution of the vertices in the limiting cases of higher rapidity resolution to the calculation of the inclusive cross-section for gluon production. One finds that this contribution is zero. In fact this result trivially follows from two circumstances. First, in the integration over longitudinal momenta of the reggeons at small $p_{ \pm}$their order of magnitude automatically reduces to the transferred momenta $\epsilon$ and $\lambda$. Then the condition of smallness of $p_{ \pm}$relative to longitudinal momenta of the reggeons transforms into smallness relative to $\epsilon$ and $\lambda$. Second, dimensional considerations restrict the final dependence of the longitudinal integrals over "-" components to either $1 / \epsilon$ or $\delta(\epsilon)$ and over "+" components to either $1 / \lambda$ or $\delta(\lambda)$. Then vanishing of the contribution in the domain (13) immediately follows.

We do not exclude cases that the obtained properties of the vertices at limiting values of gluon momenta may have other less trivial applications. We are going to search for such applications in our future study.

Data Availability Statement This manuscript has no associated data or the data will not be deposited. [Authors' comment: This article has no extra associated data.]

Open Access This article is distributed under the terms of the Creative Commons Attribution 4.0 International License (http://creativecomm ons.org/licenses/by/4.0/), which permits unrestricted use, distribution, and reproduction in any medium, provided you give appropriate credit to the original author(s) and the source, provide a link to the Creative Commons license, and indicate if changes were made.

Funded by SCOAP ${ }^{3}$.

\section{Appendix. Vertex RR $\rightarrow$ RRG in the kinematics (13)}

\subsection{Contribution from Fig. 3a}

On mass shell, multiplied by the polarization vector $e$, the corresponding amplitude $\Gamma_{1}$ is given by

$\Gamma_{1}=-C_{1} \frac{1}{t^{2} k_{1}^{2}} X_{1}, \quad X_{1}=-b \bar{B}-c \bar{C}+\mathrm{d} \bar{E}$.

The denominator is

$t^{2} k_{1}^{2}=\left(-2 p_{+} r_{1-}+t_{\perp}^{2}+i 0\right)\left(-2 q_{1+} r_{1-}+k_{1 \perp}^{2}+i 0\right)$.

The coefficients $b, c$ and $e$ are

$b=2 p_{+}\left(\left(q_{1} e\right)_{\perp}-(p e)_{\perp} \frac{q_{1+}}{p_{+}}\right)-2 q_{1+}\left(r_{2} e\right)_{\perp}$,

$c=2 p_{+}\left(\left(q_{2} e\right)_{\perp}-(p e)_{\perp} \frac{q_{2+}}{p_{+}}\right)-2 q_{2+}\left(r_{2} e\right)_{\perp}$,

$d=-2\left(p+r_{2}, e\right)_{\perp}=-2(t e)_{\perp}$.

These coefficients do not depend on $r_{1-}$, nor on $r_{2-}$. The terms $\bar{B}, \bar{C}$ and $\bar{E}$ are

$$
\begin{aligned}
\bar{B}= & -4 r_{1-}, \quad \bar{C}=-4 r_{1-}+2 \frac{r_{1}^{2}}{q_{1+}}, \\
\bar{E}= & -2 r_{1-}\left(2 q_{1+}+q_{2+}\right)+q_{1}^{2}+q_{2}^{2}-k_{1}^{2}+r_{1}^{2} \\
& +\left(a_{1}, t+q_{2}\right)_{\perp}+2 r_{1}^{2} \frac{q_{2+}}{q_{1+}}-\frac{r_{1}^{2} q_{2}^{2}}{q_{1+} r_{1-}},
\end{aligned}
$$

where $a_{1}=q_{1}+r_{1}$.

We rewrite

$X_{1}=-(b+c) \bar{B}-2 c \frac{r_{1}^{2}}{q_{1+}}+\mathrm{d} \bar{E} \equiv X_{11}+X_{12}+X_{13}$.

So

$X_{11}=-(b+c) \bar{B}=8 r_{1-} p_{+}\left(r_{1} e\right)_{\perp}$,

with $b+c=2 p_{+}\left(r_{1} e\right)_{\perp}$ Next we find

$$
\begin{aligned}
X_{12} & =-2 c \frac{r_{1}^{2}}{q_{1+}}=-4 \frac{r_{1}^{2}}{q_{1+}}\left(p_{+}\left(q_{2} e\right)_{\perp}-q_{2+}(t e)_{\perp}\right) \\
& =4 p_{+}\left(k_{1} e\right)_{\perp} \frac{r_{1}^{2}}{q_{1+}}-4 r_{1}^{2}(t e)_{\perp}
\end{aligned}
$$

and finally

$$
\begin{aligned}
X_{13}= & 2(t e)_{\perp}\left(2 r_{1-}\left(2 q_{1+}+q_{2+}\right)-q_{1}^{2}-q_{2}^{2}+r_{1}^{2}-k_{1}^{2}\right. \\
& \left.-2 p_{+} \frac{r_{1}^{2}}{q_{1+}}-\left(a_{1}, t+q_{2}\right)_{\perp}+\frac{q_{2}^{2} r_{1}^{2}}{q_{1+} r_{1-}}\right) .
\end{aligned}
$$

The leading terms in our kinematics are proportional to $1 / q_{1+} r_{1-}$. So terms in $X_{1}$ which grow more slowly than 
$p_{+} r_{1-}$ can be dropped. In particular term $X_{12}$ can be dropped altogether. Separating the longitudinal terms in $\bar{E}$ we have

$$
\begin{aligned}
\bar{E}= & -2 p_{+} r_{1-}+q_{1}^{2}+q_{2}^{2}-k_{1 \perp}^{2}-r_{1}^{2} \\
& +\left(a_{1}, t+q_{2}\right)_{\perp}+2 r_{1}^{2} \frac{p_{+}}{q_{1+}}-\frac{r_{1}^{2} q_{2}^{2}}{q_{1+} r_{1-}} .
\end{aligned}
$$

Only the first term contributes after multiplication by $d$.

Combining all terms we have

$X_{1}=4 p_{+} r_{1-}\left(e, q_{1}+q_{2}+r_{1}\right)_{\perp}$,

which gives the final result (14).

\subsection{Contribution from Fig. 3b}

On mass shell, multiplied by the polarization vector $e$, the corresponding amplitude $\Gamma_{2}$ is given by

$\Gamma_{2}=-C_{2} \frac{1}{\bar{t}^{2} k_{1}^{2}} X_{2}, \quad X_{2}=\bar{a} A+\bar{b} B+\bar{c} C+\bar{d} E$.

The denominator is

$\bar{t}^{2} k_{1}^{2}=\left(2 q_{1+} p_{-}+\bar{t}_{\perp}^{2}\right)\left(-2 q_{1+} r_{1-}+k_{1 \perp}^{2}+i 0\right)$.

The coefficients $\bar{a}, \ldots \bar{e}$ are

$$
\begin{aligned}
\bar{a}= & (p e)_{\perp} \frac{\bar{t}^{2}}{p_{+}} \text {where } \bar{t}^{2}=2 q_{1+} p_{-}+\bar{t}_{\perp}^{2}, \\
\bar{b}= & 2 p_{-}\left(r_{1} e\right)_{\perp}+2 r_{1-}\left(\left(q_{2} e\right)_{\perp}-(p e)_{\perp} \frac{q_{2+}}{p_{+}}\right) \\
& +2(p e)_{\perp}\left(r_{1-}-r_{1-} \frac{q_{2}^{2}}{p_{\perp}^{2}}+\frac{\left(p r_{1}\right)_{\perp}}{p_{+}}\right), \\
\bar{c}= & 2 p_{-}\left(r_{2} e\right)_{\perp}+2 r_{2}-\left(\left(q_{2} e\right)_{\perp}-(p e)_{\perp} \frac{q_{2+}}{p_{+}}\right) \\
& +2(p e)_{\perp}\left(r_{2-}-r_{2}-\frac{q_{2}^{2}}{p_{\perp}^{2}}+\frac{\left(p r_{2}\right)_{\perp}}{p_{+}}\right), \\
\bar{d}= & 2\left(q_{2} e\right)_{\perp}+2(p e)_{\perp}\left(1-\frac{q_{2+}}{p_{+}}-\frac{q_{2}^{2}}{p_{\perp}^{2}}\right) .
\end{aligned}
$$

Furthermore,

$$
\begin{aligned}
A= & 3 q_{1+}-\frac{q_{1}^{2}}{r_{1-}}, \quad B=4 q_{1+}, \quad C=4 q_{1+}-2 \frac{q_{1}^{2}}{r_{1-}} \\
E= & -2 q_{1+}\left(2 r_{1-}+r_{2-}\right)+r_{2}^{2}+r_{1}^{2}-k_{1}^{2} \\
& +q_{1}^{2}+2 q_{1}^{2} \frac{r_{2-}}{r_{1-}}-\left(a_{1}, \bar{t}-r_{2}\right)_{\perp}-\frac{q_{1}^{2} r_{2}^{2}}{r_{1-} q_{1+}} .
\end{aligned}
$$

We present

$$
\begin{aligned}
X_{2} & =\bar{a} A+(\bar{b}+\bar{c}) B-2 \bar{c} \frac{q_{1}^{2}}{r_{1-}}+\bar{d} E \\
& \equiv X_{21}+X_{22}+X_{23}+X_{24} .
\end{aligned}
$$

In our limit we can drop the second term in $\bar{a}$. So $X_{21}=3(p e)_{\perp} \frac{q_{1+}}{p_{+}} \bar{t}^{2}=6(p e) \frac{q_{1+}^{2} p_{-}}{p_{+}}+3(p e) \frac{q_{1+}}{p_{+}} \bar{t}_{\perp}^{2}$.

Next we find

$$
\begin{aligned}
\bar{b}+\bar{c}= & 2 p_{-}\left[\left(e q_{1}\right)_{\perp}-(e p)_{\perp} \frac{q_{1+}}{p_{+}}\right. \\
& \left.-(e p)_{\perp}\left(1-\frac{q_{2}^{2}}{p_{\perp}^{2}}+2 \frac{\left(p, r_{1}+r_{2}\right)_{\perp}}{p_{\perp}^{2}}\right)\right],
\end{aligned}
$$

so that

$$
\begin{aligned}
& X_{22}=-8(p e)_{\perp} \frac{q_{1+}^{2} p_{-}}{p_{+}} \\
& \quad+8 q_{1+} p_{-}\left[\left(e q_{1}\right)_{\perp}-(e p)_{\perp}\left(1-\frac{q_{2}^{2}}{p_{\perp}^{2}}+2 \frac{\left(p, r_{1}+r_{2}\right)_{\perp}}{p_{\perp}^{2}}\right)\right] .
\end{aligned}
$$

Next we find the terms of interest in the part $X_{23}$,

$X_{23}=4(p e)_{\perp} \frac{q_{1+}}{p_{+}} q_{1}^{2}$.

Finally, the most complicated term is $X_{24}$. Separating the longitudinal momenta in $E$ we find terms which do not vanish at $q_{1+}, r_{1-} \rightarrow \infty$

$$
\begin{aligned}
E & =2 p_{-} q_{1+}+E_{0}, \\
E_{0} & =r_{1}^{2}+r_{2}^{2}-q_{1}^{2}-k_{1 \perp}^{2}-\left(q_{1}+r_{1}, \bar{t}-r_{2}\right)_{\perp}
\end{aligned}
$$

and multiplying by $\bar{d}$ we find

$$
\begin{aligned}
X_{24}= & 4(p e)_{\perp} \frac{q_{1+}^{2} p_{-}}{p_{+}}+2(p e)_{\perp} \frac{q_{1+}}{p_{+}} E_{0} \\
& +4 q_{1+} p_{-}\left(\left(q_{2} e\right)_{\perp}-(p e)_{\perp} \frac{q_{2}^{2}}{p_{\perp}^{2}}\right) .
\end{aligned}
$$

Summing all terms we have

$$
\begin{aligned}
X_{2}= & 2(p e)_{\perp} \frac{q_{1+}^{2} p_{-}}{p_{+}}+(p e)_{\perp} \frac{q_{1+}}{p_{+}}\left(3 \bar{t}_{\perp}^{2}\right. \\
& \left.+4 p_{\perp}^{2}-2 q_{2}^{2}+4 q_{1}^{2}+8\left(p, r_{1}+r_{2}\right)_{\perp}+2 E_{0}\right) \\
& +4 q_{1+} p_{-}\left(2 q_{1}+q_{2}, e\right) .
\end{aligned}
$$

We have to take into account that the denominator $\bar{t}^{2}$ has to be taken with the next order correction,

$$
\frac{1}{\bar{t}^{2}}=\frac{1}{2 q_{1+p_{-}}+\bar{t}_{\perp}^{2}}=\frac{1}{2 q_{1+p_{-}}}\left(1-\frac{\bar{t}_{\perp}^{2}}{2 q_{1+p_{-}}}\right) .
$$

As a result

$$
\frac{X_{2}}{\bar{t}^{2} k_{1}^{2}}=-\frac{1}{4 q_{1+}^{2} r_{1-} p_{-}}\left(X_{2}-(p e) \frac{q_{1+}}{p_{+}} \bar{t}_{\perp}^{2}\right),
$$

so that we get

$\Gamma_{2}=-C_{2} \frac{\tilde{X}_{2}}{4 q_{1+}^{2} r_{1-} p_{-}}$ 
where

$$
\begin{aligned}
\tilde{X}_{2}= & 2(p e)_{\perp}+(p e)_{\perp} \frac{q_{1+}}{p_{+}}\left(2 \bar{t}_{\perp}^{2}+4 p_{\perp}^{2}-2 q_{2}^{2}+4 q_{1}^{2}\right. \\
& \left.+8\left(p . r_{1}+r_{2}\right)_{\perp}+2 E_{0}\right)+4 q_{1+} p_{-}\left(2 q_{1}+q_{2}, e\right) .
\end{aligned}
$$

$\Gamma_{2}$ can be rewritten in the form

$$
\begin{aligned}
\Gamma_{2}= & C_{2}\left\{\frac{1}{2}(p e)_{\perp} \frac{1}{p_{+} r_{1-}}-\frac{1}{q_{1+} r_{1-}}\left(e, 2 p-2 q_{1}-q_{2}\right)_{\perp}\right. \\
& \left.-\frac{1}{q_{1+} r_{1-}} \frac{(p e)_{\perp}}{p_{\perp}^{2}}\left(4\left(p, r_{1}+r_{2}\right)_{\perp}+\bar{t}_{\perp}^{2}+2 q_{1}^{2}-q_{2}^{2}+E_{0}\right)\right\} .
\end{aligned}
$$

Here $\bar{t}=p-q_{2}$.

\subsection{Contribution from Fig. 3c}

This diagram generates two terms with different color factors. The corresponding amplitudes $\Gamma_{3}$ and $\Gamma_{4}$ are given by

$\Gamma_{3,4}=C_{3,4} \frac{1}{k_{1}^{2}} X_{3,4}$,

where the denominator is $k_{1}^{2}=-2 q_{1+} r_{1-}+k_{1 \perp}^{2}+i 0$.

We have

$$
\begin{gathered}
X_{3}=-\left(a_{1} e\right)_{\perp}+2 \frac{(p e)_{\perp}}{p_{+}}\left(\frac{q_{1}^{2}}{r_{1-}}-q_{1+}\right) \\
-2 \frac{(p e)_{\perp} q_{2}^{2}}{p_{\perp}^{2} r_{2-}}\left(\frac{r_{1}^{2}}{q_{1+}}-r_{1-}\right)
\end{gathered}
$$

and

$$
\begin{gathered}
X_{4}=-\left(a_{1} e\right)_{\perp}-\frac{(p e)_{\perp}}{p_{+}}\left(\frac{q_{1}^{2}}{r_{1-}}-q_{1+}\right) \\
+\frac{(p e)_{\perp} q_{2}^{2}}{p_{+} r_{1-} r_{2-}}\left(\frac{r_{1}^{2}}{q_{1+}}-r_{1-}\right) .
\end{gathered}
$$

Here $a_{1}=q_{1}+r_{1}$.

All terms in $X_{3,4}$ which are small in the limit $q_{1+}, r_{1-} \rightarrow$ $\infty$ can be dropped. Then we obtain in a straightforward manner (16) and (17).

\subsection{Contribution from Fig. 3d}

On mass shell and convoluted with the polarization vector, the corresponding amplitude $\Gamma_{5}$ is given by

$$
\begin{aligned}
\Gamma_{5}= & C_{5} \frac{1}{k_{1}^{2} k_{2}^{2}} X_{5}, \quad X_{5}=2\left(k_{2} L_{1}\right) L_{2}-2\left(k_{1} L_{2}\right) L_{1} \\
& +\left(L_{1} L_{2}\right)\left(k_{1}-k_{2}\right)_{e} .
\end{aligned}
$$

Here $k_{1,2}=q_{1,2}-r_{1,2}$ and $C_{5}=C_{1}+C_{2}$. The denominator is

$k_{2}^{2} k_{1}^{2}=\left(-2 q_{2+} r_{2-}+k_{2 \perp}^{2}+i 0\right)\left(-2 q_{1+} r_{1-}+k_{1 \perp}^{2}+i 0\right)$.

The Lipatov vertices convoluted with polarization vectors are

$$
\begin{aligned}
L_{1} & =\left(a_{1} e\right)_{\perp}-\frac{(p e)_{\perp}}{p_{+}}\left(\frac{q_{1}^{2}}{r_{1-}}-q_{1+}\right), \\
L_{2} & =\left(a_{2} e\right)_{\perp}-\frac{(p e)_{\perp}}{p_{+}}\left(\frac{q_{2}^{2}}{r_{2-}}-q_{2+}\right), \\
\left(k_{1}-k_{2}\right)_{e} & =\left(k_{1}-k_{2}, e\right)_{\perp}-\frac{(p e)_{\perp}}{p_{+}}\left(q_{1+}-q_{2+}\right),
\end{aligned}
$$

where $a_{1}=q_{1}+r_{1}$ and $a_{2}=q_{2}+r_{2}$.

One finds

$$
\begin{aligned}
\left(k_{2} L_{1}\right)= & \left(p L_{1}\right)=-p_{+} r_{1-}-p_{-} q_{1+} \\
& +\left(p a_{1}\right)_{\perp}+r_{1}^{2} \frac{p_{+}}{q_{1+}}+q_{1}^{2} \frac{p_{-}}{r_{1-}}, \\
\left(k_{1} L_{2}\right)= & \left(p L_{2}\right)=-p_{+} r_{2-}-p_{-} q_{2+} \\
& +\left(p a_{2}\right)_{\perp}+r_{2}^{2} \frac{p_{+}}{q_{2+}}+q_{2}^{2} \frac{p_{-}}{r_{2-}},
\end{aligned}
$$

and finally

$$
\begin{aligned}
\left(L_{1} L_{2}\right)= & \left(a_{1} a_{2}\right)_{\perp}+q_{1+} r_{2-}+q_{2+} r_{1-}-r_{1}^{2} \frac{q_{2+}}{q_{1+}} \\
& -r_{2}^{2} \frac{q_{1+}}{q_{2+}}-q_{1}^{2} \frac{r_{2-}}{r_{1-}}-q_{2}^{2} \frac{r_{1-}}{r_{2-}}+\frac{q_{1}^{2} r_{2}^{2}}{r_{1-} q_{2+}}+\frac{q_{2}^{2} r_{1}^{2}}{r_{2-} q_{1+}} .
\end{aligned}
$$

In our limit we get

$$
\begin{aligned}
& L_{2}\left(k_{2} L_{1}\right)=(p e)_{\perp} \frac{q_{2+}}{p_{+}}\left(-p_{+} r_{1-}-p_{-} q_{1+}\right) \\
& \quad+(p e)_{\perp} \frac{q_{2+}}{p_{+}}\left(p a_{1}\right)_{\perp}+\left(a_{2} e\right)_{\perp}\left(-p_{+} r_{1-}-p_{-} q_{1+}\right)
\end{aligned}
$$

and

$$
\begin{aligned}
& L_{1}\left(k_{1} L_{2}\right)=(p e)_{\perp} \frac{q_{1+}}{p_{+}}\left(-p_{+} r_{2-}-p_{-} q_{2+}\right) \\
& \quad+(p e)_{\perp} \frac{q_{1+}}{p_{+}}\left(p a_{2}\right)_{\perp}+\left(a_{1} e\right)_{\perp}\left(-p_{+} r_{2-}-p_{-} q_{2+}\right) .
\end{aligned}
$$

Taking the difference we find

$$
\begin{aligned}
& L_{2}\left(k_{2} L_{1}\right)-L_{1}\left(k_{1} L_{2}\right) \\
& =(p e)_{\perp}\left(q_{1+} r_{2-}-q_{2+} r_{1-}\right) \\
& \quad+(p e)_{\perp} \frac{1}{p_{+}}\left(q_{2+}\left(p a_{1}\right)_{\perp}-q_{1+}\left(p a_{2}\right)_{\perp}\right) \\
& \quad+\left(a_{2} e\right)_{\perp}\left(-p_{+} r_{1-}-p_{-} q_{1+}\right) \\
& \quad-\left(a_{1} e\right)_{\perp}\left(-p_{+} r_{2-}-p_{-} q_{2+}\right) .
\end{aligned}
$$

Only terms quadratic in $q_{1+}=-q_{2+}$ and $r_{1-}=-r_{2-}$ can give a non-zero contribution in our limit. However, as we see, they are canceled in the first term. So we do not get any contribution from the first two terms in $X_{5}$ 
In the third term we can leave only the leading term in $\left(L_{1} L_{2}\right)$

$\left(L_{1} L_{2}\right)=q_{1+} r_{2-}+q_{2+} r_{1-}$,

which leads to

$$
\begin{aligned}
X_{5}= & -\frac{(p e)_{\perp}}{p_{+}}\left(q_{1+}-q_{2+}\right)\left(q_{1+} r_{2-}+q_{2+} r_{1-}\right) \\
& +\left(k_{1}-k_{2}, e\right)_{\perp}\left(q_{1+} r_{2-}+q_{2+} r_{1-}\right) .
\end{aligned}
$$

In the second term we can take $q_{2+}=-q_{1+}$ and $r_{2-}=$ $-r_{1}-$ to finally get

$$
\begin{aligned}
\Gamma_{5}= & C_{5}\left\{\frac{1}{2} \frac{(p e)_{\perp}}{p_{+}}\left(\frac{1}{r_{1-}}-\frac{1}{r_{2-}}\right)\right. \\
& \left.-\frac{1}{2 q_{1+} r_{1-}}\left(q_{1}-r_{1}-q_{2}+r_{2}, e\right)_{\perp}\right\} .
\end{aligned}
$$

Note that the first term is the only one where the difference between $r_{1-}$ and $-r_{2-}$ is significant.

\section{References}

1. L.V. Gribov, E.M. Levin, M.G. Ryskin, Phys. Lett. B 121, 65 (1983)

2. Yu.V. Kovchegov, K. Tuchin, Phys. Rev. D 65, 074026 (2002)
3. E.A. Kuraev, L.N. Lipatov, V.S. Fadin, Sov. Phys. JETP 45, 199 (1977)

4. I.I. Balitski, L.N. Lipatov, Sov. J. Nucl. Phys. 28, 822 (1978)

5. J. Bartels, Nucl. Phys. B 151, 293 (1979)

6. M.A. Braun, Eur. Phys. J. C 48, 501 (2006)

7. K. Dusling, F. Gelis, T. Lappi, R. Venugopalan, Nucl. Phys. A 836, $159(2010)$

8. L.N. Lipatov, Phys. Rep. 286, 1997 (1997)

9. V.S. Fadin, L.N. Lipatov, Phys. Lett. B 429, 127 (1998)

10. M. Hentschinski, A. Sabio Vera, Phys. Rev. D 85 (2012) 056006; arXiv: 1110.6741 [hep-th]

11. G. Chachamis, M. Hentschinski, J.D. Madrigal Martinez, A. Sabio Vera, Phys. Rev. D 87, 076009 (2013). arXiv:1212.4992 [hep-th]

12. G. Chachamis, M. Hentschinski, J.D. Madrigal Martinez, A. Sabio Vera, Nucl. Phys. B 876, 453 (2013). arXiv:1307.2591 [hep-th]

13. M. Nefedov, V. Saleev, Mod. Phys. Lett A 32, 1750207 (2017). arXiv:1709.06246 [hep-th]

14. V.A. Abramovsky, V.N. Gribov, O.V. Kancheli, Sov. J. Nucl. Phys. 18, 308 (1974)

15. M.A. Braun, M.I. Vyazovsky, Eur. Phys. J. C 51, 103 (2007)

16. M.A. Braun, L.N. Lipatov, M.Yu. Salykin, M.I. Vyazovsky, Eur. Phys. J. C 71, 1639 (2011)

17. M.A. Braun, S.S. Pozdnyakov, M.Yu. Salykin, M.I. Vyazovsky, Eur. Phys. J. C 72, 2223 (2012)

18. M.A. Braun, S.S. Pozdnyakov, M.Yu. Salykin, M.I. Vyazovsky, Eur. Phys. J. C 75, 222 (2015) 